\title{
Biometric and Forensic Aspects of Digital Document Processing
}

\author{
Sargur N. Srihari, Chen Huang, Harish Srinivasan, and Vivek Shah \\ Center of Excellence for Document Analysis and Recognition (CEDAR) \\ University at Buffalo, State University of New York \\ 520 Lee Entrance, Amherst, NY 14228, USA \\ \{srihari\}@cedar.buffalo.edu
}

\begin{abstract}
Signatures and handwriting have long played a role in dayto-day business transactions and in forensics, e.g., to authenticate documents, as evidence to establish crime or innocence, etc. The individuality of handwriting and signatures is the basis for their relevance to authentication and forensics. This very individuality makes them also potentially useful as a biometric modality. This chapter is concerned with automatic methods for verifying the writership of handwritten documents and signatures. The discussion consists of the individuality of handwriting, image pre-processing and interactive tools for forensic document examination, discriminating characteristics of handwriting, a statistical model of writer verification, and application of the model to signature verification.
\end{abstract}

\section{Introduction}

The field of Forensic Document Examination [FDE] is concerned with issues such as whether the writer of a questioned document, say a ransom note, is the same as the known writer of sample documents, whether a signature is genuine or is a forgery, etc. The basis of the use of handwriting as evidence is its individuality, i.e., every person's writing is different, or every person's signature is unique.

More recently researchers in biometrics have been developing automated means for authenticating a person, i.e., verifying whether a person is indeed who he/she claims to be. The commonly considered modalities for biometrics are fingerprints, iris, hand-prints, voice, gait, etc. Since handwriting is a characteristic that is potentially unique to each individual and since it can be captured non-invasively, handwriting and signatures can also be useful as biometrics.

The field of FDE is much broader than the examination of handwriting and signatures. For instance, the examination of inks, typed and printed manuscripts are also in the purview of FDE. The area of commonality between forensics and biometrics is the examination of handwriting and signature by automated methods. It is this area of intersection which is addressed in this chapter.

Forensic examination has been largely based on manual examination by the expert to discern writing habits and other characteristics. Automated methods for handwriting examination are only recently being developed and introduced 
to the FDE community. Automated signature versification has a longer history although much of the work has been in the on-line case. The use of handwriting and signatures in biometrics is still more recent. Since handwriting is a behavioral characteristic, in contrast to biological characteristics such as fingerprints, handwriting is possibly useful to biometrics only when used in conjunction with other modalities.

\subsection{Individuality of Handwriting}

Writer identification has a long history perhaps dating to the origins of handwriting itself. Classic forensic handwriting examination is primarily based upon the knowledge and experience of the forensic expert.

The individuality of handwriting has been a contentious issue in the courts. There are several rulings in the United States courts that are concerned with the admissibility of handwriting as evidence. The central ruling on this issue is the supreme court case of Daubert versus Merrell Dow Pharamceuticals which required that any expert evidence to be admitted has to have a scientific basis. Whether a theory or methodology has a scientific basis has many philosophical implications, e.g., whether it is falsifiable. Four specific creteria to determine whether there exists a scientific basis for an expertise were proposed as follows: (i) experimentation, (ii) error rates, (iii) peer review of the methods, and (iv) general acceptance.

Since many types of evidence used by the courts, e.g., fingerprints and handwriting, did not have support in all four measures research was only recently undertaken to fill the gaps. The individuality of handwriting was studied recently leading to a characterization of the individuality of handwriting when there were sufficient amount of data available[1].

Due to the subjective nature of expert decisions, traditional methods are being supplemented by computerized semi-automatic and interactive systems. Such systems allow for large-scale testing so that error rates can be determined. The choice of the test sets is relevant, e.g., testing on data from twins or other cohort types would pose a more challenging test than when they are collected otherwise.

\subsection{Organization of Chapter}

Section 2 describes image pre-processing operations and interactive user interfaces for FDE. Section 3 describes discriminating elements, also known as features or characteristics, that are useful for writer/signature discrimination. Section 4 describes a statistical model for writer verification. This model is also applicable to signature verification and other biometric modalities such as voice and fingerprints. Section 5 describes an approach to signature verification which includes performance in terms of false acceptance rates and false rejection rates. The concluding remarks in Section 6 indicates the future of handwriting in forensics and biometrics. 


\section{Image Pre-processing and Interactive Tools}

A computer system for retrieving a small set of documents from a large set, known as the Forensic Information System for Handwriting, or the FISH system [2], has been developed by German law enforcement. Also motivated by the forensic application, a handwritten document management system, known as CEDAR-FOX [3], has been developed for handwritten document analysis, identification, verification and document retrieval. As a document management system for forensic analysis, CEDAR-FOX provides user three major functionalities. First it can be used as a document analysis system. Second it can be used for creating a digital library for forensic handwritten documents and third it can be used as a database management system for document retrieval and writer identification. The CEDAR-FOX system is used as a case study in the rest of this paper.

As an interactive document analysis system a graphic interface is provided. It can scan or load a handwritten document image. The system will first automatically extract features based on document image processing and recognition. The user can then use tools provided to perform document examination and extract document metrics. These tools include image selection, image enhancement, contour display, etc.

\subsection{Region of Interest (ROI) Selection}

A document image may include many text or graphical objects. In a complex document there could be combinations of machine-printed (or typed) paragraphs, tables, accompanying annotations, logos and signatures. The user often needs to specify a local region of most interest. A cropping tool is provided so that user can scissor-out a region of interest (ROI) from the original document and then do all the analysis based on the selected ROI.

In addition, forensic document examiners use writing characteristic pertaining to certain characters or glyphs in comparing documents. For example, one of the features they often look for is the lower loop in characters "g" and " $\mathrm{y}$ ". Thus identification and recognition of document components including those belonging to the same character category are always necessary and important. Besides automatic identification and recognition for isolated handwritten characters, the system also provides a useful tool for document examiners to easily crop out certain character images manually. Then a set of features will be computed for the cropped characters for comparison. Figure 1 shows a screenshot of manually defined characters. The upper image shows a letter image "g" manually selected from each of two documents below.

\subsection{Image Pre-processing}

Several tools for pre-processing the image need to be available for the purpose of preparing the image for analysis, either for further automatic processing or for human visual examination. Some of these, viz., thresholding, rule-line removal, contour display, and stroke thickening are described here. 


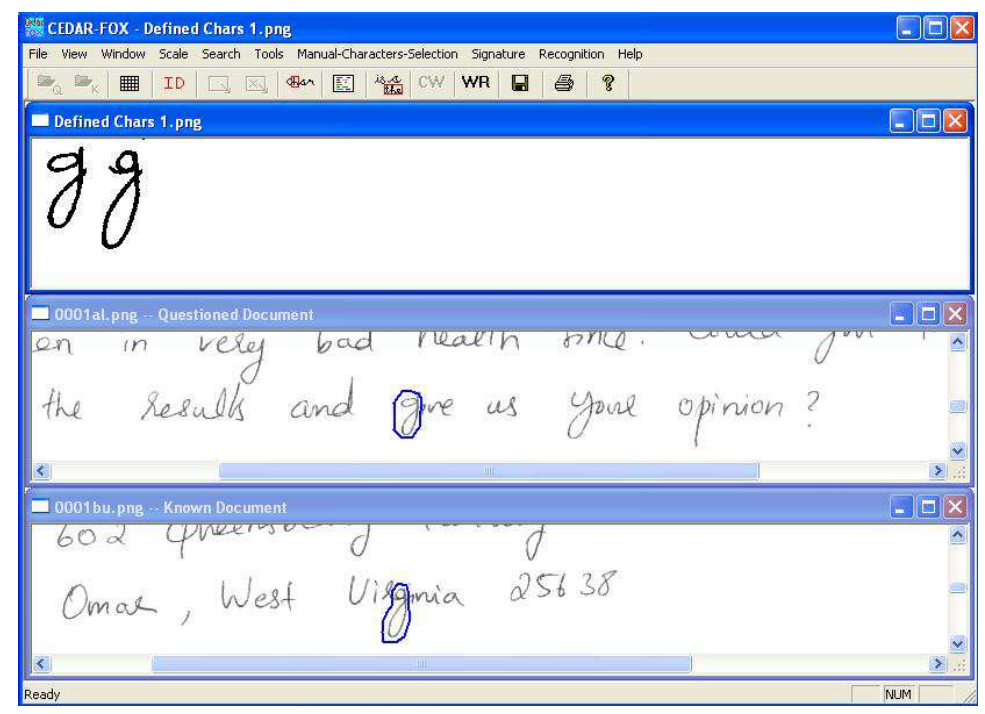

Fig. 1. Manually defined characters selected using an interactive device, e.g., mouse.

Thresholding: Thresholding is to convert a gray scale image into binary by determining a value for gray-scale (or threshold) below which the pixel can be considered to belong to the writing and above which to the background. The operation is useful to separate the foreground layer of the image, i.e., the writing, from the back-ground layer, i.e., the paper. The system includes several types of thresholding algorithms, e.g., global thresholding- when the background is uniform as in the case of a cleanly written sheet of paper, adaptive thresholdingwhen the contrast varies. Figure 2 shows the result of a thresholding operation.

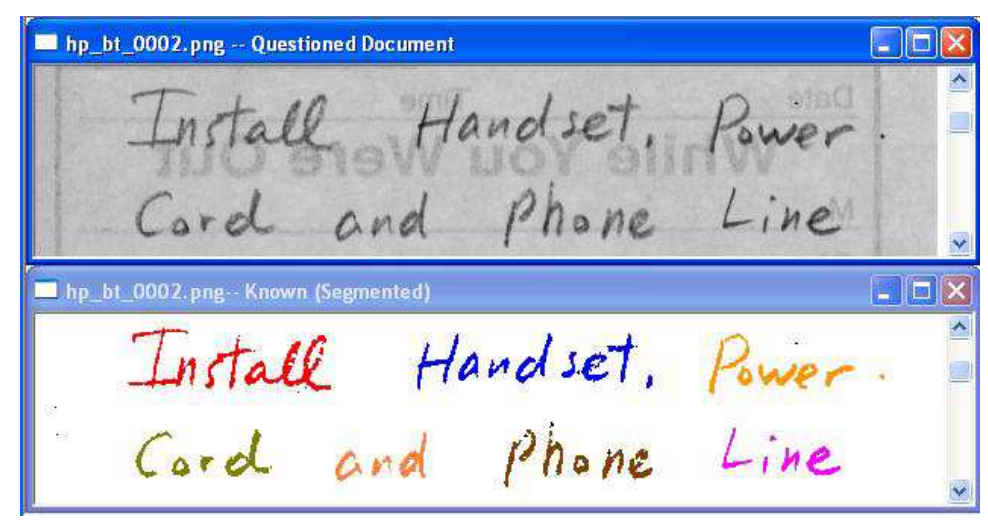

Fig. 2. Thresholding a gray-scale image into a binary image followed by line and word segmentation. 
Segmentation: Once the foreground image is obtained the writing can then be segmented, or scissored, into lines and words of text. Figure 2 shows the segmented lines and words also.

Underline Removal: The use of ruled-line paper is common in handwritten documents. Words and phrases are also sometimes underlined. If the document was written using rule-lined paper, an 'underline removal' operation will erase the underlines automatically. Figure 3 shows a screen-shot of underline removal operation.

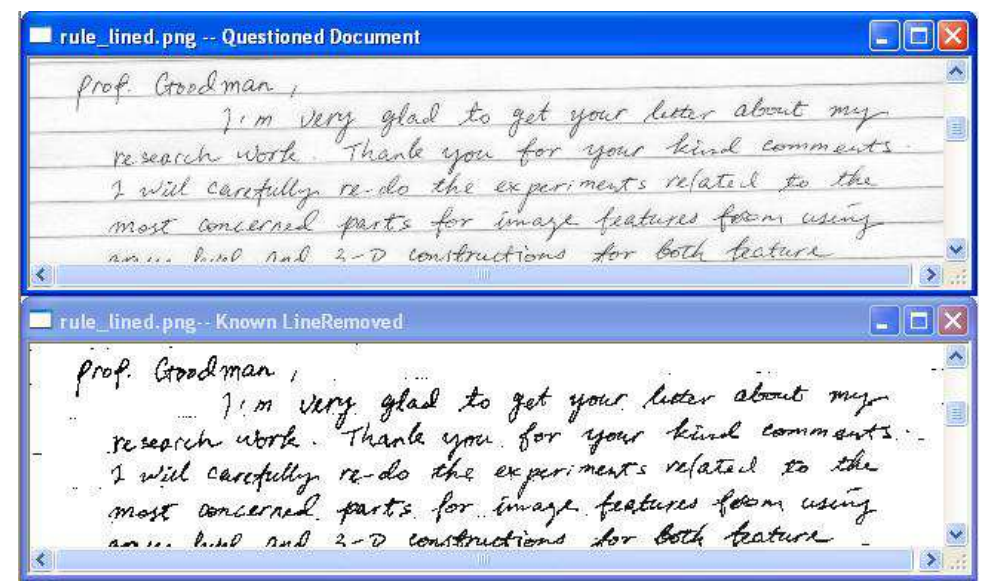

Fig. 3. Removal of ruled-lines.

Contour Display: For the purpose of visual examination by a document examiner, displaying the detailed inner and outer contours of each stroke are useful. Figure 4 shows such a contour display.

Stroke Thickening: This operation takes faint lines and thickens them for visibility, which is shown in Figure 5.

\section{Discriminating Elements and their Similarities}

Discriminating elements are characteristics of handwriting useful for writer discrimination. There are many discriminating elements for FDE, e.g., there are 21 classes of discriminating elements [4]. In order to match elements between two documents, the presence of the elements are first recognized in each document. Matching is performed between the same elements in each document. 


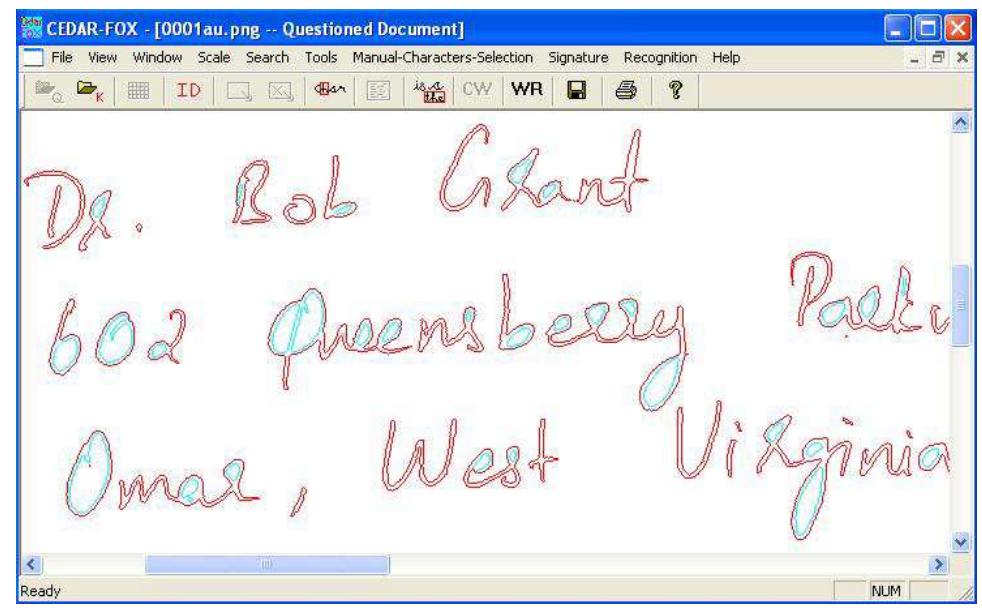

Fig. 4. Contour display.

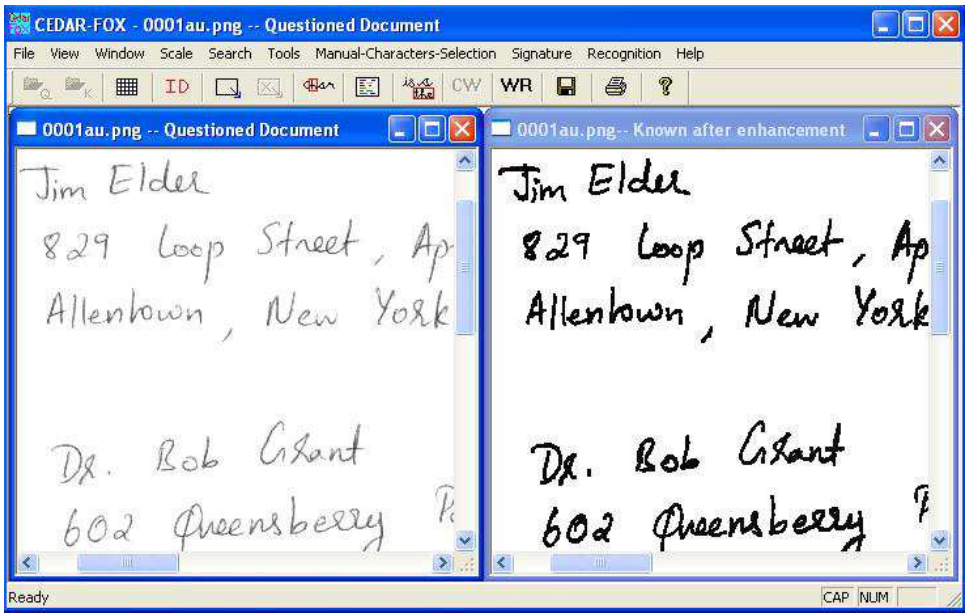

Fig. 5. Stroke thickening to enhance visibility.

Features that capture the global characteristics of the writer's individual writing habit and style can be regarded to be macro-features and features that capture finer details at the character level as micro-features. For instance macro features are gray-scale based (entropy of the distribution of gray-scale values in the document, the threshold needed to separate foreground from background (discussed in Section 2.2), and the number of black pixels in the document after thresholding), contour based (external and internal contours), slope-based (horizontal, positive, vertical and negative), stroke-width, slant and height. Details of these features are described in [1]. 
Micro features are attributes that describe the shapes of individual characters. A set of micro-features that capture the finest variations in the contour, intermediate stroke information and larger concavities and enclosed regions, e.g., [1] are derived from the scanned image of a character. These features are obtained from the character image by first imposing a $4 \times 4$ grid on it. From this grid a 512-bit feature vector is determined as follows. Gradient (G) features, which are obtained using an image gradient (or derivative) operator, measure the magnitude of change in a $3 \times 3$ neighborhood of each pixel. For each grid cell, by counting statistics of 12 gradients, there are $4 \times 4 \times 12=192$ gradient features. Structural (S) features capture certain patterns, i.e., mini-strokes, embedded in the gradient map. A set of 12 rules is applied to each pixel- to capture lines, diagonal rising and corners- yielding 192 structural features. Concavity (C) features, which capture global and topological features, e.g., bays and holes, are $4 \times 4 \times 8=128$ in number. These features were previously used in character recognition [5], word recognition [6] and writer identification [1]. The concept of micro features can also be expanded to pairs of characters and other glyphs.

\subsection{Similarity}

Since macro features are real-valued the absolute difference of the values for two documents can be used as the distance measure (or measure of dissimilarity).

Since micro features are binary-valued several binary string distance measures can be used for similarity of characters, the most effective of which is the correlation measure [7]. In order to match characters it is first necesary to segment the character from the document and know its character-class, i.e, whether it is the character a or b, etc. This can be done either by automatic character recognition or by providing the truth and segmentation manually.

Similarity histograms corresponding to the same writer and different writer distributions for the numeral 3 (micro feature) and for entropy (macro feature) are shown in Figure 6.

\section{Writer Verification}

Writer verification is the task of determining whether two handwriting samples were written by the same or by different writers. In contrast, the task of writer identification is to determine for a questioned document as to which individual, with known handwriting, it belongs to. Identification can be accomplished by repeated verification against each known individual. However a higher accuracy identification method can be devised by taking advantage of the differences among the known writers.

This section describes a statistical model of the task of verification which has three salient components: (i) parametric modeling of probability densities of feature dissimilarities, conditioned on being from the same or different writer, (ii) design of the statistical classifier and (iii) computing the strength of evidence. Each of the components of the model are described in the following sections. 

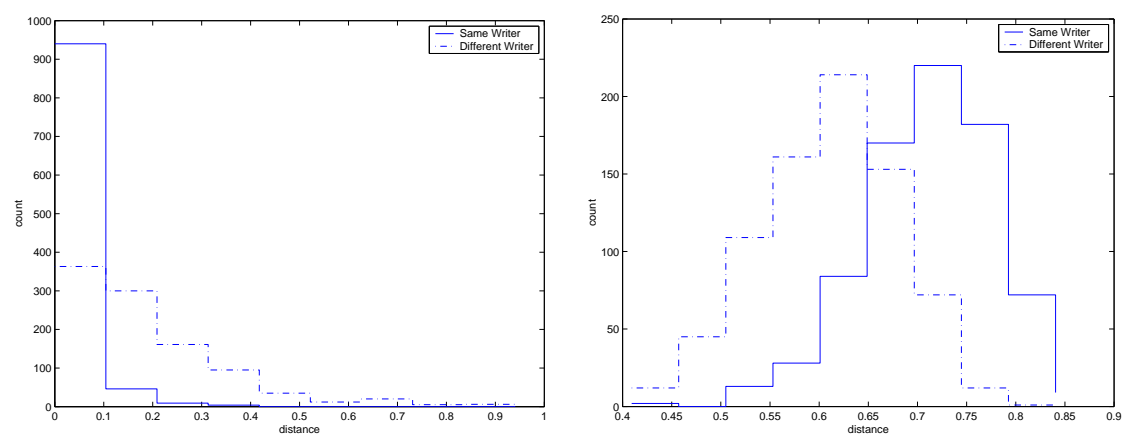

Fig. 6. Histograms of dissimilarity between pairs of handwriting samples for same and different writers for: (a) entropy, which is a macro feature, and (b) numeral 3, which is characterized by micro features.

\subsection{Parametric Models}

The distributions of distances (or dissimilarities) conditioned on being from the same or different writer are useful to determine whether a given pair of samples belong to the same writer or not. In the case of similarities that are continuousvalued it is useful to model them as parametric densities- since it only involves storing the distribution parameters. Several choices exist for the parametric forms of the densities, e.g., Gaussian or other exponential distributions such as the gamma distribution.

Assuming that the similarity data can be acceptably represented by Gaussian or gamma distributions, the probability density functions of distances conditioned upon the same-writer and different-writer categories for a single feature $x$ have the parametric forms $p_{s}(x) \sim N\left(\mu_{s}, \sigma_{s}^{2}\right), p_{d}(x) \sim N\left(\mu_{d}, \sigma_{d}^{2}\right)$ for the Gaussian case, and $p_{s}(x) \sim \operatorname{Gam}\left(a_{s}, b_{s}\right), p_{d}(x) \sim \operatorname{Gam}\left(a_{d}, b_{d}\right)$ for the gamma case. These parameters are estimated using maximum likelihood estimates. The Gaussian and gamma density functions are as follows:

$$
\begin{gathered}
\text { Gaussian }: p(x)=\frac{1}{(2 \pi)^{1 / 2} \sigma} \exp ^{-\frac{1}{2}\left(\frac{x-\mu}{\sigma}\right)^{2}} \\
\text { Gamma }: p(x)=\frac{x^{a-1} \exp ^{(-x / b)}}{(\Gamma(a)) \cdot b^{a}}
\end{gathered}
$$

Conditional parametric pdfs for the numeral 3 (micro-feature) and for entropy (macro feature) are shown in Figure 7.

The Kullback Leibler (KL) divergence test was performed for each of the features to estimate whether it is better to model them as Gaussian or as gamma distributions. The gamma distribution is possibly the better model since distances are positive-valued whereas the Gaussian assigns non-zero probabilities to negative values of distances. Table 1 gives the KL test result values, in bits, for each macro feature. A training set size of 1000 samples was chosen for each of 

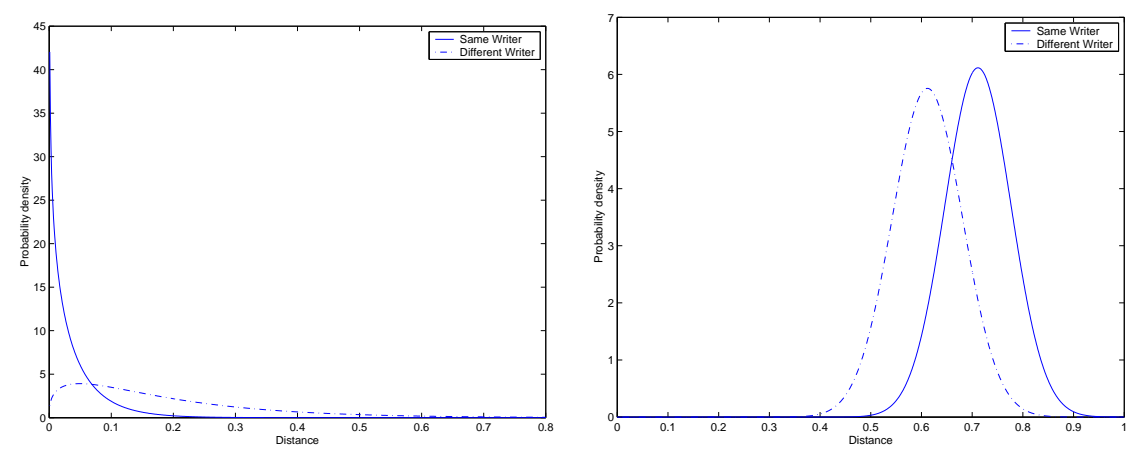

Fig. 7. Parametric pdfs for: (a) distances along the entropy feature, which are modeled by gamma distributions, and (b) distances between pairs of numeral 3s which are modeled by Gaussian distributions.

same and different writers. The test set size was 500 for each. As can be seen the gamma values are consistently lower than that of the Gaussian values thereby indicating that the gamma is a better fit.

Table 1. KL test results for the 12 macro features.

\begin{tabular}{|c||c|c||c|c|}
\hline \multirow{2}{*}{ Macro Feature } & \multicolumn{2}{c||}{ Same Writer } & \multicolumn{2}{c|}{ Different Writer } \\
\cline { 2 - 5 } & Gamma & Normal & Gamma & Normal \\
\hline Entropy & 0.133 & 0.921 & 0.047 & 0.458 \\
\hline Threshold & 3.714 & 4.756 & 2.435 & 3.882 \\
\hline No. Black Pixels & 1.464 & 2.314 & 2.151 & 2.510 \\
\hline External contours & 2.421 & 3.517 & 2.297 & 2.584 \\
\hline Internal contours & 2.962 & 3.373 & 2.353 & 2.745 \\
\hline Horizontal Slope & 0.050 & 0.650 & 0.052 & 0.532 \\
\hline Positive Slope & 0.388 & 1.333 & 0.173 & 0.315 \\
\hline Vertical Slope & 0.064 & 0.664 & 0.054 & 0.400 \\
\hline Negative Slope & 0.423 & 1.385 & 0.113 & 0.457 \\
\hline Stroke width & 3.462 & 6.252 & 3.901 & 4.894 \\
\hline Average Slant & 0.392 & 1.359 & 0.210 & 0.362 \\
\hline Average Height & 3.649 & 4.405 & 2.558 & 2.910 \\
\hline
\end{tabular}

The parameters of the distributions of the macro feature distances (for a training set of size 1000) are given in Table 2.

The likelihood ratio (LR) summarizes the statement of the comparison process. The $\mathrm{LR}$ values for a given $\mathrm{x}$ (where $\mathrm{x}$ is the random variable) is obtained as $p_{s}(x) / p_{d}(x)$. The log-likelihood ratio (LLR), obtained by taking the logarithm of LR, is more useful since LR values tend to be very large (or small). The error rates (percent misclassification) obtained from a classifier based on the LLR (evaluated for each macro feature, using a test size of 500) are given in Table 3. 
Table 2. Gaussian and gamma parameters for the 12 macro features.

\begin{tabular}{|l||c|c|c|c||c|c|c|c|}
\hline \multirow{2}{*}{\multicolumn{1}{|c||}{ Feature }} & \multicolumn{4}{c||}{ Gaussian Parameters } & \multicolumn{3}{c|}{ Gamma Parameters } \\
\cline { 2 - 10 } & Same Writer & Different Writer & Same Writer & \multicolumn{2}{c|}{ Different Writer } \\
\cline { 2 - 10 } & mean & std & mean & std & a & b & a & b \\
\hline Entropy & 0.0379 & 0.044 & 0.189 & 0.162 & 0.752 & 0.050 & 1.355 & 0.139 \\
\hline Threshold & 1.603 & 2.025 & 12.581 & 35.430 & 0.627 & 2.559 & 0.126 & 99.779 \\
\hline No. Black Pixels & 22761 & 28971 & 107061 & 89729 & 0.617 & 36875 & 1.424 & 75204 \\
\hline External contours & 1.828 & 2.965 & 9.135 & 7.703 & 0.380 & 4.810 & 1.406 & 6.496 \\
\hline Internal contours & 2.626 & 2.348 & 5.830 & 5.144 & 1.251 & 2.100 & 1.285 & 4.538 \\
\hline Horizontal Slope & 0.013 & 0.014 & 0.072 & 0.066 & 0.930 & 0.014 & 1.179 & 0.061 \\
\hline Positive Slope & 0.014 & 0.023 & 0.112 & 0.081 & 0.392 & 0.037 & 1.890 & 0.059 \\
\hline Vertical Slope & 0.016 & 0.016 & 0.101 & 0.083 & 1.041 & 0.015 & 1.492 & 0.068 \\
\hline Negative Slope & 0.008 & 0.013 & 0.060 & 0.050 & 0.381 & 0.021 & 1.416 & 0.042 \\
\hline Stroke width & 0.235 & 0.431 & 0.968 & 1.185 & 0.297 & 0.791 & 0.667 & 1.451 \\
\hline Average Slant & 1.730 & 2.674 & 12.402 & 8.955 & 0.419 & 4.133 & 1.918 & 6.465 \\
\hline Average Height & 1.809 & 1.920 & 8.458 & 7.147 & 0.888 & 2.037 & 1.400 & 6.039 \\
\hline
\end{tabular}

The average error rate is lower for gamma over Gaussian although for one of the two classes (same writer) the Gaussian does better.

Table 3. Comparison of error rates for each macro feature when distances are modeled using gamma and Gaussian distributions.

\begin{tabular}{|c||c|c||c|c|}
\hline \multicolumn{1}{|c||}{ Macro Feature } & \multicolumn{2}{c||}{ Same Writer } & \multicolumn{2}{c|}{ Different Writer } \\
\cline { 2 - 5 } & Gamma & Normal & Gamma & Normal \\
\hline Entropy & 21.30 & 13.00 & 23.20 & 38.40 \\
\hline Threshold & 2.60 & 2.60 & 53.40 & 60.00 \\
\hline No. Black Pixels & 22.19 & 9.80 & 22.40 & 39.60 \\
\hline External contours & 30.10 & 6.20 & 18.60 & 46.80 \\
\hline Internal contours & 28.30 & 8.80 & 33.00 & 56.60 \\
\hline Horizontal Slope & 13.44 & 5.40 & 25.20 & 34.40 \\
\hline Positive Slope & 10.59 & 3.60 & 16.80 & 31.20 \\
\hline Vertical Slope & 11.60 & 5.60 & 23.20 & 31.60 \\
\hline Negative Slope & 14.46 & 3.00 & 23.10 & 37.00 \\
\hline Stroke width & 23.20 & 23.20 & 0.00 & 31.60 \\
\hline Average Slant & 9.97 & 3.00 & 18.60 & 31.40 \\
\hline Average Height & 17.43 & 5.00 & 22.40 & 40.80 \\
\hline
\end{tabular}

\subsection{Design of Classifier}

Since the document is characterized by more than one feature we need a method of combining the feature values. We assume that the writing elements are statistically independent. Although this is strictly incorrect the assumption has 
a certain robustness in that it is not an overfitting of the data. The resulting classifer, also known as the Naive Bayes classifier, has yielded good results in machine learning. Moreover, in the earliest FDE literature, there is reference to multiplying the probabilities of handwriting elements e.g., [8].

Each of the two likelihoods that the given pair of documents were either written by the same or different individuals can be expressed, assuming statistical independence of the features as follows. For each writing element $e_{i}, i=1, \ldots, c$, where $\mathrm{c}$ is the number of writing elements considered, we compute the distance $d_{i}(j, k)$ between the jth occurrence of $e_{i}$ in the first document and the kth occurrence of $e_{i}$ in the second document for that writing element. We estimate the likelihoods as

$$
\begin{aligned}
L_{s} & =\prod_{i=1}^{c} \prod_{j} \prod_{k} p_{s}\left(d_{i}(j, k)\right) \\
L_{d} & =\prod_{i=1}^{c} \prod_{j} \prod_{k} p_{d}\left(d_{i}(j, k)\right)
\end{aligned}
$$

The log-likelihood ratio (LLR) in this case has the form

$$
L L R=\sum_{i=1}^{c} \sum_{j} \sum_{k}\left[\ln p_{s}\left(d_{i}(j, k)\right)-\ln p_{d}\left(d_{i}(j, k)\right)\right]
$$

The two cumulative distributions of $L L R$ s corresponding to same and different writer samples are shown in Figure 8 (a) and (b). It is observed that as the number of features considered decreases the separation between the two curves also decreases. The separation gives an indication of the separability between classes. The more the separation the easier it is to classify.
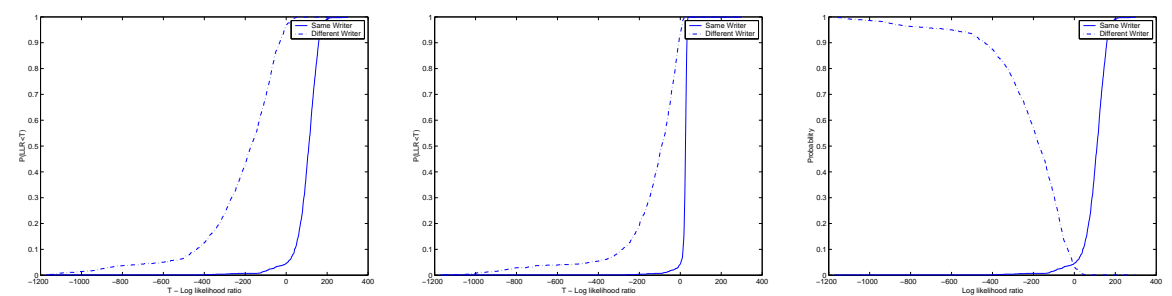

Fig. 8. CDF of LLRs for same and different writer populations (a) 12 macro and 62 micro features (b) 12 macro and 8 micro features (c) CDF of LLRs for same writer population and inverse CDF for different writer population

For macro features which are discrete, distances are modeled with a probability table. For macro features which are continuous-valued they are modeled as gamma distributions. The list of features and the distributions they are modelled by is given in Table 4 . The list of macro features modelled as gamma and their parameters are given in Table 5 . 
Table 4. Statistical Models for for macro and micro features

\begin{tabular}{|c|c|c|}
\hline Feature & Type of Distribution & Modeled using \\
\hline Entropy & Continuous & Gamma \\
\hline Threshold & Discrete & Probability table \\
\hline Black Pixels & Continuous & Gamma \\
\hline Exterior contours & Discrete & Probability table \\
\hline Interior contour & Discrete & Probability table \\
\hline Horizontal slope & Continuous & Gamma \\
\hline Positive slope & Continuous & Gamma \\
\hline Vertical Slope & Continuous & Gamma \\
\hline Negative slope & Continuous & Gamma \\
\hline Stroke width & Discrete & Probability table \\
\hline Avg. slant & Discrete & Probability table \\
\hline Avg. height & Continuous & Gamma \\
\hline Avg. word Gap & Continuous & Gamma \\
\hline $\begin{array}{c}\text { Micro features } \\
(0-9, \text { A-Z,a-z })\end{array}$ & Continuous & Gamma \\
\hline
\end{tabular}

Table 5. Gamma parameters for continuous macro features.

\begin{tabular}{|c|c|c|c|c|}
\hline \multirow{2}{*}{ Feature } & \multicolumn{2}{|c|}{ Shape parameter } & \multicolumn{2}{c|}{ Scale parameter } \\
\cline { 2 - 5 } & Different writer & Same writer & Different writer & Same writer \\
\hline Entropy & 2.3585 & 2.7967 & 0.0179 & 0.0149 \\
\hline Black Pixels & 1.4838 & 1.7143 & 3520 & 1736.6 \\
\hline Horizontal slope & 1.6041 & 1.2237 & 0.0444 & 0.0199 \\
\hline Positive slope & 1.8346 & 0.9005 & 0.0569 & 0.0187 \\
\hline Vertical Slope & 1.8138 & 1.5775 & 0.0523 & 0.0150 \\
\hline Negative slope & 1.3219 & 0.9506 & 0.0426 & 0.0100 \\
\hline Avg. height & 1.5134 & 1.3562 & 5.2860 & 2.0184 \\
\hline Avg. word Gap & 2.9363 & 3.4487 & 0.0372 & 0.0128 \\
\hline
\end{tabular}

The distributions of micro feature distances are also modeled as gamma distributions.

Performance with Twins Data Set: In many areas of forensics it is traditional to evaluate performance on a set of twins. This is because they presumably pose higher similarities than a general population.

Automatic writer verification was performed on a data-set consisting of handwriting samples obtained from more than two hundred pairs of twins. This was done for both micro and macro features using both the gamma and Gaussian parametric models. It was found that gamma performed better (94.7\%) than Gaussian (93.5\%) for macro features but did not improve the results for micro features. This was more evident in the case of non-twins than twins. Considering just the macro features we were able to get $94.7 \%$ accuracy for samples of non 
twins. A similar test was also done on the twin data set and found that gamma and Gaussian error rate are similar. Table 6 gives the error rate for twins and non twins for each of the macro features. The system was trained using 1500 same and 1500 different writer samples of different content.

Table 6. Macro feature performance with twins and non-twins sample pairs.

\begin{tabular}{|l||c|c||c|c||c|c|}
\hline \multirow{2}{*}{$\begin{array}{c}\text { Features under } \\
\text { Consideration }\end{array}$} & \multicolumn{2}{c||}{$\begin{array}{c}\text { Same Writer } \\
\text { Different content(SD) }\end{array}$} & \multicolumn{2}{c||c|}{$\begin{array}{c}\text { Different Writer } \\
\text { Different content(DD) }\end{array}$} & $\begin{array}{c}\text { Average error rate } \\
\text { SD+DD }\end{array}$ \\
\cline { 2 - 6 } & Twins & Non-Twins & Twins & Non-Twins & Twins & Non-Twins \\
\hline Entropy & $45.7 \%$ & $34.7 \%$ & $40.1 \%$ & $45.6 \%$ & $42.9 \%$ & $40.0 \%$ \\
\hline Threshold & $18.0 \%$ & $21.4 \%$ & $36.9 \%$ & $33.7 \%$ & $27.5 \%$ & $27.6 \%$ \\
\hline Black Pixels & $32.8 \%$ & $30.0 \%$ & $41.8 \%$ & $35.6 \%$ & $37.3 \%$ & $32.8 \%$ \\
\hline Exterior contours & $22.9 \%$ & $30.0 \%$ & $49.1 \%$ & $41.6 \%$ & $36.0 \%$ & $35.8 \%$ \\
\hline Interior contour & $35.5 \%$ & $32.5 \%$ & $44.3 \%$ & $43.8 \%$ & $39.9 \%$ & $38.1 \%$ \\
\hline Horizontal slope & $20.2 \%$ & $23.8 \%$ & $42.1 \%$ & $27.3 \%$ & $31.2 \%$ & $25.5 \%$ \\
\hline Positive slope & $13.6 \%$ & $13.2 \%$ & $35.5 \%$ & $23.6 \%$ & $24.6 \%$ & $18.4 \%$ \\
\hline Vertical Slope & $17.5 \%$ & $16.5 \%$ & $41.4 \%$ & $24.0 \%$ & $29.5 \%$ & $20.2 \%$ \\
\hline Negative slope & $14.5 \%$ & $14.9 \%$ & $38.9 \%$ & $28.4 \%$ & $26.7 \%$ & $21.6 \%$ \\
\hline Stroke width & $31.9 \%$ & $31.3 \%$ & $41.1 \%$ & $36.5 \%$ & $36.5 \%$ & $33.9 \%$ \\
\hline Avg. slant & $15.6 \%$ & $12.0 \%$ & $36.7 \%$ & $23.0 \%$ & $26.2 \%$ & $17.5 \%$ \\
\hline Avg. height & $24.8 \%$ & $27.8 \%$ & $45.7 \%$ & $37.7 \%$ & $35.3 \%$ & $32.7 \%$ \\
\hline Avg. word Gap & $22.4 \%$ & $19.8 \%$ & $38.4 \%$ & $29.1 \%$ & $30.4 \%$ & $24.4 \%$ \\
\hline
\end{tabular}

\subsection{Evaluating the strength of evidence}

In order to present the result in the form of the strength of evidence [9], it is useful to represent the $L L R$ scores on a scale ranging from -1 to 1 ( -1 representing a confident different writer case and 1 representing a confident same writer case). It would be inappropriate to state all results with $L L R>0$ as same writer and all results with $L L R<0$ as different writer. Instead of using binary decisions a range of interpreted results is always better and more practical from the QDE point of view. Based on the Tippet plot [10] (inverse CDF plot) and the CDF plot we develop a scheme for calibrating the $L L R$ scores. The CDF and inverse $\mathrm{CDF}$ for same and different writer $L L R \mathrm{~s}$ (considering 12 macro features and 62 micro features) is shown in Figure 8 (c).

In the case of characters since the number of matches is variable resulting in multiple instances of each feature. Since the number of features is unbound it is necessary to do some averaging to bound the value. Assume a set of $\mathrm{m}$ features (macro features and 62 characters $0-9, a-z, A-Z$ represented by micro features). If for the $i^{\text {th }}$ feature we get $k_{i} L L R$ values, $L L R_{i 1}, L L R_{i 2}, . L L R_{i k_{i}}$ then their average is 


$$
L L R_{\text {ave }}(i)=\frac{1}{k_{i}} \sum_{j=1}^{k_{i}} L L R_{i j}
$$

For each feature $i$ the CDF and inverse CDF for same and different writers are obtained from the distribution of $L L R_{\text {ave }}(i)$. For the same writer case we obtain $P_{\text {same }_{i}}\left(L L R<L L R_{\text {ave }}(i)\right)$ from the CDF of same writer $L L R$ for that feature. For the different writer case we obtain $P_{\text {diff }}\left(L L R>L L R_{\text {ave }}(i)\right)$ from the inverse CDF of different writer $L L R$ for that feature.

Assuming $m$ features are available we compute the geometric means

$$
P 1=\prod_{j=1}^{m}\left(P_{\text {same }_{j}}\right)^{1 / m} \text { and } P 2=\prod_{j=1}^{m}\left(P_{\text {diff }}\right)^{1 / m}
$$

to make the calibration independent of the number of features present. Finally we compute the calibration score as Score $=P 1-P 2$.

The values of Score is always between -1 and 1. QD examiners use nine categories of opinion: identify, highly probable, probable, indicative did, no conclusion, indicative did not, probably did not, highly probabe did not and eliminate. The scatter plots of scores obtained are shown in Figure 9 for 500 for same and 500 different writer cases. Observing the histograms of scores for same and different writers the score range is divided into nine zones.

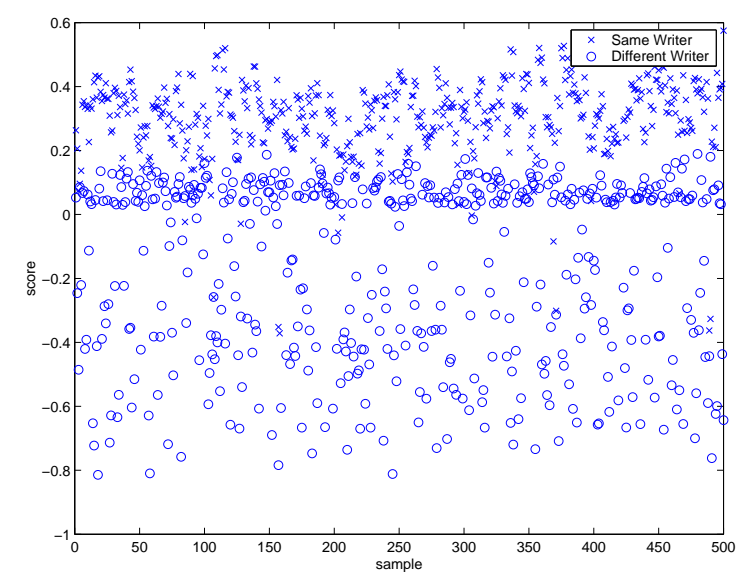

Fig. 9. Scatter plots of scores obtained for same and different writer test set after calibration

Table 7 summarizes the results of the calibration on the test set. The first four zones are same writer zones, the fifth is 'no conclusion' zone and the last four zones are different writer zones. Based on the zones obtained $2.2 \%$ of same writer and $4.8 \%$ of different writer test cases fell into the 'no conclusion' zone. Same 
writer accuracy obtained was $94.6 \%$ while different writer accuracy is $97.6 \%$. Same writer accuracy is obtained as the ratio of the number of same writer test cases falling into same writer zones to the total number of same writer test cases not falling into the 'no conclusion' zone. Similarly different writer accuracy is obtained as the ratio of the number of different writer test cases falling into different writer zones to the total number of different writer test cases not falling into the 'no conclusion' zone.

Table 7. Strength of evidence calibration of $L L R$ values (for the test set of 500 same and 500 different)

\begin{tabular}{|c|c|c|}
\hline Category & $\begin{array}{c}\text { Same Writer } \\
(\% \text { in each category })\end{array}$ & $\begin{array}{c}\text { Different Writer } \\
(\% \text { in each category })\end{array}$ \\
\hline Identified as same $(>0.5)$ & 2.0 & 0.0 \\
\hline Highly probable same $(>0.35 \&<0.5)$ & 34.6 & 0.0 \\
\hline Probably did $(>0.2 \&<0.35)$ & 48.8 & 0.0 \\
\hline Indications Did $(>0.15 \&<0.2)$ & 7.2 & 4.2 \\
\hline No Conclusion $(>0.12 \&<0.15)$ & 2.2 & 45.4 \\
\hline Indications did not $(>-0.05 \&<0.12)$ & 3.6 & 12.6 \\
\hline Probably did not $(>-0.3 \&<-0.05)$ & 0.6 & 27.8 \\
\hline Highly probable did not $(>-0.65 \&<-0.3)$ & 1.0 & 7.2 \\
\hline Identified as different $(<-0.65)$ & 0.0 & \\
\hline
\end{tabular}

\subsection{Summary of Writer Verification}

A statistical model for a handwriting verification has been described. First a set of characteristics from the questioned and known documents are extracted and their corresponding differences are recorded. The likelihoods for the two classes are computed assuming statistical independence of the distances- where the conditional probabilities for the differences are estimated using parametric probability densities which are either Gaussian or gamma. The log-likelihood ratio $(L L R)$ of same and different writer are computed for decision-making. Cumulative distributions of the $L L R$ s are used to calibrate the $L L R$ values so as to present the strength of evidence.

Using a specific set of characteristics with the model, same writer accuracy was $94.6 \%$ and different writer accuracy was $97.6 \%$. Overall accuracy was $96.1 \%$ with only $3.5 \%$ of test cases falling into the 'no conclusion' zone.

\section{Signature Verification}

Automatic verification of signatures from scanned paper documents has many applications such as authentication of bank checks, questioned document examination, biometrics, etc. On-line, or dynamic, signature verification systems have 
been reported with high success rates [11]. However, off-line, or static research is relatively unexplored- which difference can be attributed to the lack of temporal information, the range of intra-personal variation in the scanned image, etc.

Methods have been described for both writer-dependent (WD) and writerindependent (WI) signature verification. WD models extract features from genuine signatures of a specific writer and are trained for that writer. The questioned signature is compared against the model for that writer. This is the standard approach to signature verification [12]. Based on a writer-independent approach to determining whether two handwritten documents- not just signatures- were written by the same person or not [1], a writer independent (WI) signature verification method was proposed in [13]. In the WI model the probability distributions of within-writer and between-writer similarities, over all writers, are computed in the training phase. These distributions are used to determine the likelihood of whether a questioned signature is authentic.

\subsection{Learning Strategies}

Signature verification is a problem that can be approached using machine learning techniques. A set of samples of signatures, $D$, can be prepared with the help of several individuals. The parameters derived from such a set can be used in determining whether an arbitrary pair of signatures, e.g., a questioned signature and a genuine signature, match or not. One can also learn from samples of a specific individual and use only these parameters (or model) in matching for that individual.

These two learning strategies are: writer independent (WI) and writer dependent (WD), as shown in Figure 10. In WI learning, $D_{g}$ and $D_{f}$ are the training data sets of genuine and forged signatures from several writers. A model $S$ is trained from pairs of samples (genuine-genuine and genuine-forgery) from $D_{g}$ and $D_{f}$. Given a questioned signature $Q$ and a set $K$ of genuine signatures for individual $w, S$ is used to determine whether $Q$ is accepted as genuine. In WD learning, only the genuines for individual $w$, i.e., the set $K$, is used to determine the model $S$, which is then used to determine whether $Q$ is accepted as genuine.

\section{$5.2 \quad$ Signature Test-Bed}

A database of off-line signatures was prepared as a test bed. Each of 55 individuals contributed 24 signatures- thereby creating 1320 genuine signatures. Some were asked to forge three other writers' signatures, eight times per subject, thus creating 1320 forgeries. One example of each of 55 genuines are shown in Figure 11. Ten examples of genuines of one subject (subject no. 21) and ten forgeries of that subject are shown in Figure 12.

Image Preprocessing Each signature was scanned at 300 dpi gray-scale and binarized using a gray-scale histogram. Salt-and-pepper noise was removed by median filtering. Slant normalization was performed by extracting the axis of 


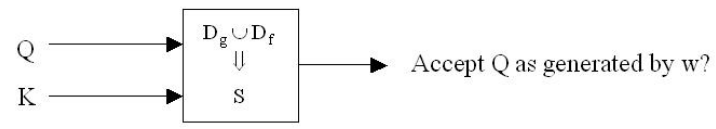

(a)

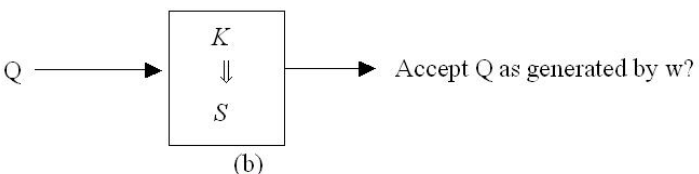

Fig. 10. Verification Models: (a) writer independent: a questioned $(Q)$ is matched against a set of genuines $K$ using a model $S$ derived from genuines and forgeries of other writers, and (b) writer dependent: a model for an individual is determined using only $K$.

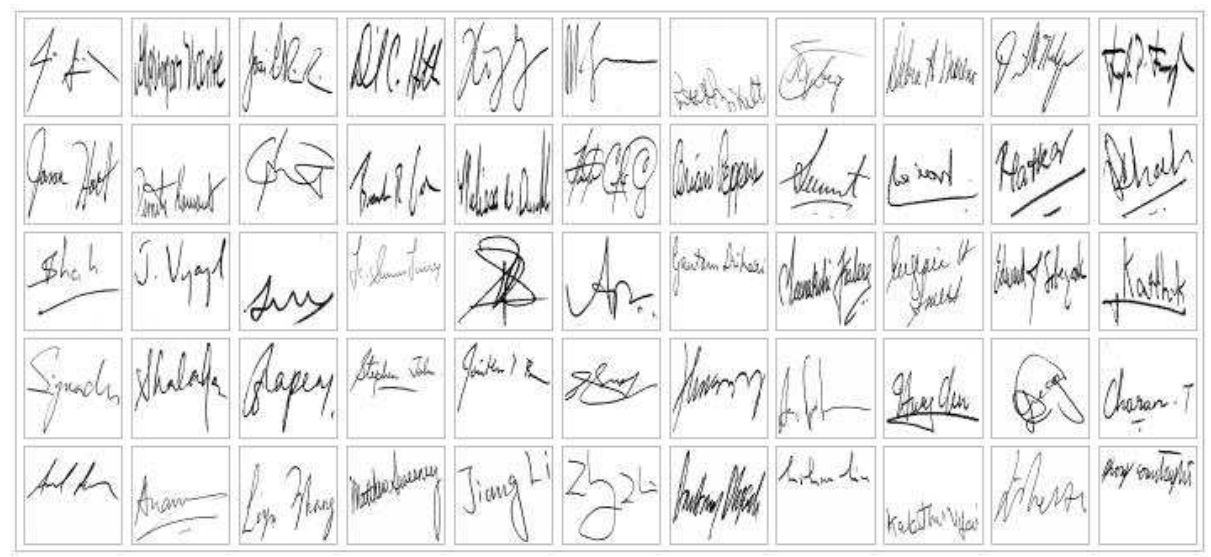

Fig. 11. Genuine signature samples.

least inertia and rotating the curve until this axis coincides with the horizontal axis [14]. Given an $M \times N$ image, $G=\left(u_{k}, v_{k}\right)=\left(x_{(i, j)}, y_{(i, j)} \mid x_{(i, j)} \neq 0, y_{(i, j)} \neq 0\right)$. Let $S$ be the size of $G$, and let $\bar{u}=\frac{1}{S} \sum_{k} u_{k}$ and $\bar{v}=\frac{1}{S} \sum_{k} v_{k}$ be the coordinates of the center of mass of the signature. The orientation of the axis of least inertia is given by the orientation of the least eigenvector of the $2 \times 2$ matrix $I=\left(\begin{array}{l}\overline{u^{2}} \\ \overline{u v} \\ \overline{v^{2}}\end{array}\right)$ where $\overline{u^{2}}=\frac{1}{S} \sum_{k}\left(u_{k}-\bar{u}\right)^{2}, \overline{v^{2}}=\frac{1}{S} \sum_{k}\left(v_{k}-\bar{v}\right)^{2}$ and $\overline{u v}=\frac{1}{S} \sum_{k}\left(u_{k}-\bar{u}\right)\left(v_{k}-\bar{v}\right)$ are the second order moments of the signature [15]. The result of binarization and slant normalization of a gray-scale image is shown in Figure 13.

Feature Extraction: Features for static signature verification can be one of three types $[16,17]$ : (i) global: extracted from every pixel that lie within a rectan- 


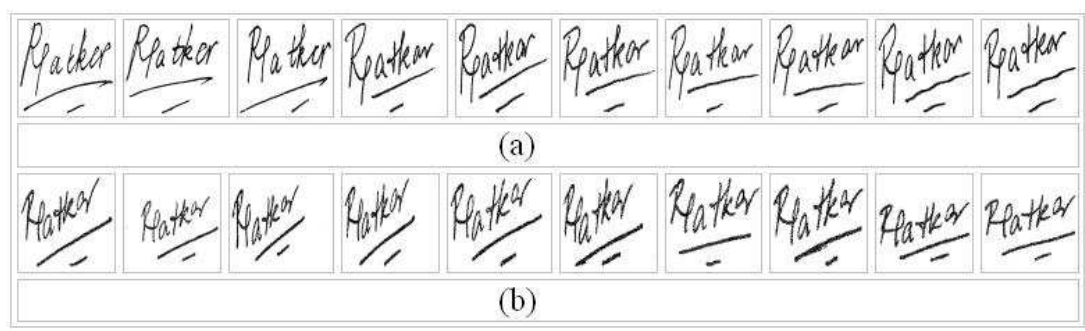

Fig. 12. Samples for one writer: (a) genuines and (b) forgeries.

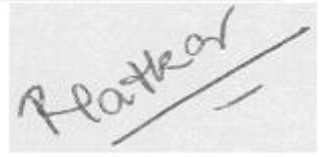

(a)

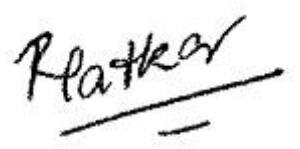

(b)

Fig. 13. Pre-processing: (a) original (b) final.

gle circumscribing the signature, including image gradient analysis [18], series expansions [19], etc., (ii) statistical: derived from the distribution of pixels of a signature, e.g., statistics of high gray-level pixels to identify pseudo-dynamic characteristics [20], (iii) geometrical and topological: e.g., local correspondence of stroke segments to trace signatures [21], feature tracks and stroke positions [16], etc. A combination of all three types of features were used in a writer independent (WI) signature verification system [13,22]. These features, expanded from the GSC features of characters (see Section 3)were used here.

The average size of all reference signature images was chosen as the reference size to which all signatures were resized. The image is divided into a $4 \times 8$ grid from which a 1024-bit GSC feature vector is determined (Figure 14). Gradient (G) features measure the magnitude of change in a $3 \times 3$ neighborhood of each pixel. For each grid cell, by counting statistics of 12 gradients, there are $4 \times 8 \times 12=384$ gradient features. Structural (S) features capture certain patterns, i.e., ministrokes, embedded in the gradient map. A set of 12 rules is applied to each pixelto capture lines, diagonal rising and corners- yielding 384 structural features. Concavity (C) features, which capture global and topological features, e.g., bays and holes, are $4 \times 8 \times 8=256$ in number.

Distance Measure: A method of measuring the similarity or distance between two signatures in feature space is essential for classification. The correlation distance performed best for GSC binary features [23] which is defined for two binary vectors $X$ and $Y$, as follows:

$$
d(X, Y)=\frac{1}{2}-\frac{s_{11} s_{00}-s_{10} s_{01}}{2\left(\left(s_{10}+s_{11}\right)\left(s_{01}+s_{00}\right)\left(s_{11}+s_{01}\right)\left(s_{00}+s_{10}\right)\right)^{1 / 2}}
$$




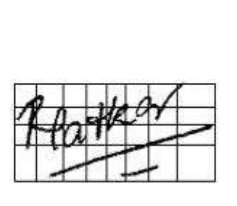

(a)

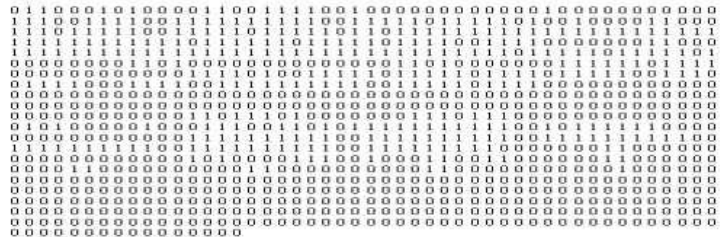

(b)

Fig. 14. Features: (a) variable grid, and (b) feature vector.

where $s_{i j}$ represent the number of corresponding bits of $X$ and $Y$ that have values $i$ and $j$. Both the WI-DS and WD-DT methods described below use $d(X, Y)$ as the distance measure.

\subsection{Writer-independent Verification}

The objective is to determine whether pair $(K, Q)$ belongs to the same individual, where $Q$ is a questioned signature and $K$ is a set of known signatures for that individual. Two WI classification methods, distance statistics [1] and naive Bayes, are presented below.

Distance Statistics (DS) Method: The verification approach of [1]is based on two distributions of distances $d(X, Y)$ : genuine-genuine and genuine-forgery pairs. The distributions are denoted as $P_{g}$ and $P_{f}$ respectively. The means and variances of $d(X, Y)$ where $X$ and $Y$ are both genuine and $X$ is genuine and $Y$ is a forgery are shown in Figure 15 - where the the number of writers varies from 10 to 55 . Here 16 genuines are 16 forgeries were randomly chosen from each subject. For each $n$, there are two values corresponding to the mean and variance of $n \times C_{2}^{16}$ pairs of same writer (or genuine-genuine pair) distances and $n \times 16^{2}$ pairs of different writer (or genuine-forgery pair) distances. The values are close to constant with $\mu_{g}=0.24$ and $\mu_{f}=0.28$ with corresponding variances $\sigma_{g}=0.055$ and $\sigma_{f}=0.05$. Given a questioned signature $Q$ and a single known signature $K$, the probabilities of $d(K, Q)$ are: $P($ genuine $\mid Q)=P_{g}(d(K, Q))$ and $P($ forged $\mid Q)=P_{f}(d(K, Q)) . Q$ is accepted as genuine if the genuine probability exceeds the forgery probability. Normal distributions are assumed for genuinegenuine distances and genuine-forgery distances.

Generalization to $n$ genuines: when there are $\mathrm{n}$ genuine signatures available, i.e., $|K|>1$, given a questioned signature $Q$,

$$
\begin{aligned}
& P(\text { genuine } \mid Q)=\prod_{j=1}^{n} P_{g}\left(d\left(K_{j}, Q\right)\right) \\
& P(\text { forged } \mid Q)=\prod_{j=1}^{n} P_{f}\left(d\left(K_{j}, Q\right)\right)
\end{aligned}
$$



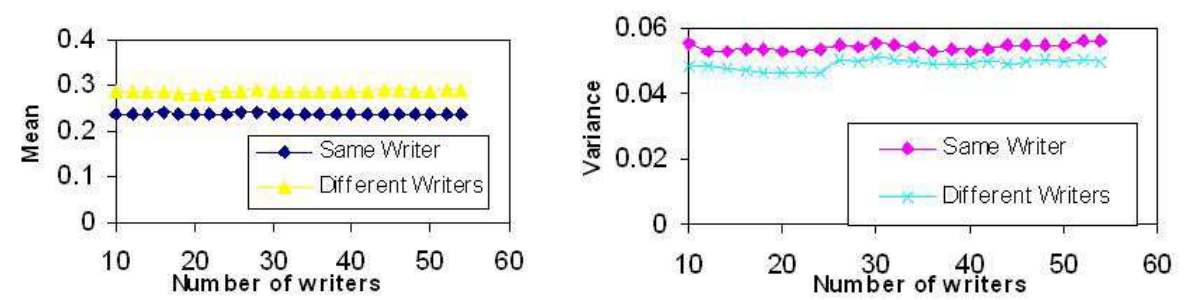

Fig. 15. Statistics of genuine-genuine and genuine-forgery distances.

Naive Bayes (NB) Method: Rather than determining the distributions of distances between two feature vectors, each pair of corresponding bits in the questioned and known feature vectors can be treated as random variables. The pairs corresponding to different positions in the feature vector are considered to be independent and identically distributed, which is the naive Bayes (NB) assumption. Let feature vectors $X=\left\{x_{1}, x_{2}, \ldots, x_{n}\right\}$ and $Y=\left\{y_{1}, y_{2}, \ldots, y_{n}\right\}$. The probabilities of $i^{t h}$ same-value bits in a genuine-genuine pair and a genuineforgery pair are computed using:

$$
\begin{gathered}
P_{s, x_{i}=y_{i}}=\frac{|(X, Y)| x_{i}=y_{i}, X, Y \in D_{g} \mid}{|(X, Y)| X, Y \in D_{g} \mid} \\
P_{s, x_{i} \neq y_{i}}=\frac{|(X, Y)| x_{i} \neq y_{i}, X, Y \in D_{g} \mid}{|(X, Y)| X, Y \in D_{g} \mid} \\
P_{d, x_{i}=y_{i}}=\frac{|(X, Y)| x_{i}=y_{i}, X \in D_{g}, Y \in D_{f} \mid}{|(X, Y)| X \in D_{g}, Y \in D_{f} \mid} \\
P_{d, x_{i} \neq y_{i}}=\frac{|(X, Y)| x_{i} \neq y_{i}, X \in D_{g}, Y \in D_{f} \mid}{|(X, Y)| X \in D_{g}, Y \in D_{f} \mid}
\end{gathered}
$$

where $D_{g}$ and $D_{f}$ are the training sets of genuine and forged signatures. Knowing the probabilities of the values of the bit pair for each feature, given $(K, Q)$, the overall genuine-genuine and genuine-forgery probabilities are calculated as the product of the probabilities for all feature pairs, i.e.,

$$
\begin{gathered}
P(\text { genuine } \mid Q)=P_{s}(K, Q)=\prod_{i=1}^{1024} P_{s, k_{i}=q_{i}}^{k_{i} \otimes q_{i}} P_{s, k_{i} \neq q_{i}}^{k_{i} \oplus q_{i}} \\
P(\text { forged } \mid Q)=P_{d}(K, Q)=\prod_{i=1}^{1024} P_{d, k_{i}=q_{i}}^{k_{i} \otimes q_{i}} P_{d, k_{i} \neq q_{i}}^{k_{i} \oplus q_{i}}
\end{gathered}
$$

They are compared to determine whether they are from the same writer or not. Generalization to $\mathrm{n}$ genuines is as follows:

$$
P(\text { genuine } \mid Q)=\prod_{j=1}^{n} P_{s}\left(K_{j}, Q\right)
$$




$$
P(\text { forged } \mid Q)=\prod_{j=1}^{n} P_{d}\left(K_{j}, Q\right)
$$

Performance: The two writer independent methods were evaluated using the test bed. False reject rate (FRR), false accept rate (FAR) and average error rate $(\mathrm{AER}=(\mathrm{FRR}+\mathrm{FAR}) / 2)$ were determined. To calculate probabilities 16 genuines and 16 forgeries from each subject were randomly chosen as the training set and the rest are used as test set. FRR, FAR and AER of two methods are shown in Table 8.

Table 8. Writer-independent methods with 1 and 16 training samples

\begin{tabular}{|l|r|r|r|}
\hline Methods(n) & FRR(\%) & FAR(\%) & AER(\%) \\
\hline Distance Stats(1) & 27.6 & 27.8 & 27.7 \\
\hline Naive Bayes(1) & 27.2 & 26.0 & 26.6 \\
\hline Distance Stats(16) & 21.3 & 22.1 & 21.7 \\
\hline Naive Bayes(16) & 22.9 & 24.1 & 23.5 \\
\hline
\end{tabular}

The probabilities in WI-DS and feature probabilities in WI-NB, the product of 16 distance probabilities in WI-DS or the product of feature p. The WIDS and WI-NB were evaluated with 16 genuines in training compared to one in Section 5.3. Instead of original distance probabilities in WI-DS and feature probabilities in WI-NB, the product of 16 distance probabilities in WI-DS or the product of feature probabilities in WI-NB were used.

With both methods performance increases with more training genuines. For training, $n$ genuines were randomly chosen. The test set consisted of 8 genuines from the rest and 24 forgeries. WI-DS has the best performance. Figure 16 shows performance improvement of WI-DS with $n$.

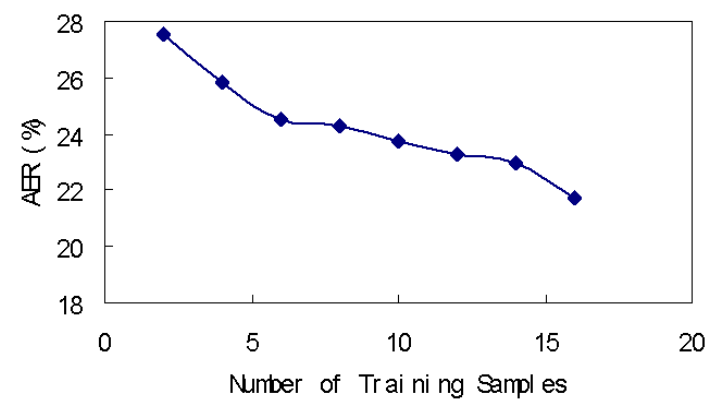

Fig. 16. Average Error Rate of Writer Independent Distance Statistics method. 


\subsection{Writer-dependent Verification}

Assuming that there exist sufficient training genuines, a machine of $S$ is learned only from the training data for a specific individual. Four classification methods were considered: distance threshold (which is the standard method used of biometrics), distance statistics, naive Bayes and SVM. Two sub-formulations are considered: one-class where forgeries for the individual are unavailable, and two-class where genuines and forgeries are available.

Training with Genuines only: Distance Threshold (DT): The DT method is the common signature verification model. The first step is to enroll genuines $K$ as reference signatures. The distance $d(X, Y)$ is computed for each pair $(X, Y)$ in $K$ to determine the threshold thres $=\max \{d(X, Y) \mid X, Y \in K\}$. Given a questioned signature $Q$, the average of $\{d(Q, Y) \mid Y \in K\}$, denoted as dist, is computed. If dist $<$ thres, then $Q$ is accepted as a genuine; and rejected otherwise.

Distance Statistics (DS): Here the genuine-genuine distance distribution is obtained only from $K$, i.e., the mean and variance of $P_{g}$ are determined from $\{d(X, Y) \mid X, Y \in K\}$. The genuine-forgery distance distribution $P_{f}$ is from $D_{g}$ and $D_{f}$ as in WI-DS.

Naive Bayes (NB): Let $X=\left\{x_{1}, x_{2}, \ldots, x_{n}\right\}$ where $X \in K$. Two distributions are computed from $K$ : Given a test signature $Q=\left\{q_{1}, q_{2}, \ldots, q_{n}\right\}$, the likelihood, $P($ genuine $\mid Q)=\prod_{i=1}^{n} P_{s, x_{i}=q_{i}}$. A common optimal threshold thres for the likelihoods is trained for all writers. $Q$ is accepted as a genuine if $P($ genuine $\mid Q) \geq$ thres.

Experimental results for three methods, with a training set size of 16 , are shown in Table 9. Here the distance threshold performs best.

Table 9. One-class writer-dependent methods (trained with genuines only)

\begin{tabular}{|l|r|r|r|}
\hline Methods(n) & FRR(\%) & FAR(\%) & AER(\%) \\
\hline Distance Threshold & 22.5 & 19.5 & 21.5 \\
\hline Distance Statistics & 23.0 & 21.7 & 22.4 \\
\hline Naive Bayes & 25.9 & 24.1 & 25.0 \\
\hline
\end{tabular}

Training with Genuines and Forgeries: Forgeries were included in training in the following experiments. In WD-DT, since the threshold is determined only by genuines, it is unchanged. However in WD-DS, instead of gathering genuine-forgery distance distribution from all writers, such distribution is generated directly from the genuine-forgery pairs for the individual. Similarly in WD-NB, 0 and 1 distributions of each feature in forgeries are generated from the feature vectors in the forgery set. 
In a support vector machine (SVM) [24, 25], hyperplanes are determined by support vectors instead of training samples. Due to unbalanced training datasets, instead of finding equal size maximal margin on both sizes of optimal hyperplanes, margins are dynamically adjusted according to sample sizes. With more positive samples than negative samples, different penalty parameters are used to balance weights. Given training vectors $x_{i} \in R^{n}, i=1, \ldots, l$, in two classes, and a vector $y \in R^{l}$ such that $y_{i} \in\{1,-1\}$, the formation in [26] solves the classification problem for unbalanced data.

For each writer, 16 genuines were randomly selected from the genuine set and 5 forgeries selected from the forgery set by one forger. The other 8 genuines and 16 forgeries by other forgers constitute the test set. Table 10 presents the classification results showing that SVM outperforms others.

Table 10. Two-class writer-dependent methods (with 16 genuines and 5 forgeries)

\begin{tabular}{|l|r|r|r|}
\hline Methods(n) & FRR(\%) & FAR(\%) & AER(\%) \\
\hline Distance Statistics & 17.6 & 20.7 & 19.2 \\
\hline Naive Bayes & 9.95 & 13.0 & 11.45 \\
\hline SVM & 8.5 & 10.1 & 9.3 \\
\hline
\end{tabular}

\subsection{Summary of Signature Verification}

Several learning strategies for signature verification were evaluated using a highdimensional feature space that captures both local geometric information as well as stroke information. In the writer-independent case, the newly introduced distance statistics method outperformed classical distance threshold and naive Bayes approaches. In the writer dependent case distance threhold performed best with distance statistics being close. The distance statistics method has the advantage that it can be used when few training examples, even one, are available, and it generates a match likelihood rather than a distance score.

\section{Concluding Remarks}

The use of automated methods in writer verification and identification is in its early stages. The use of handwriting and signatures in biometrics is at an even earlier stage. The early results indicate their potential for use in both biometrics and forensics.

Writer identification has significant potential since high accuracies can be obtained as larger samples of handwriting, e.g., line, paragraph, page, etc., are available. In the case of signatures the same high accuracies that can be obtained in the on-line case cannot be expected in the off-line case. Also it can be argued 
that since the amount of writing available in a signature is limited, compared with a paragraph of writing, its accuracies will be lower. However the signature is known to be deliberately individualistic - and therefore has a strong potential as a biometric.

The entire field of off-line handwriting processing by computer is in its infancy although much success has been achieved in constrained domains such as postal address reading and bank check reading. As recognition techniques improve the methods of writer verification will also improve, particularly since recognition is frequently the first step in verification. Improvement of recognition will in turn depend upon the ability to exploit contextual knowledge.

\section{Acknowledgement}

This project was supported in part by Grant Number 2004-IJ-CX-K050 awarded by the National Institute of Justice, Office of Justice Programs, US Department of Justice. Points of view in this document are those of the authors and do not necessarily represent the official position or policies of the US Department of Justice.

\section{References}

1. S. N. Srihari, S. Cha, H. Arora, and S. Lee, "Individuality of handwriting," Journal of Forensic Sciences, vol. 47, no. 4, pp. 856-872, July 2002.

2. K. Franke, L. Schomaker, L. Vuurpijl, and S. Giesler, "Fish-new: A common ground for computer-based forensic writer identification," Proceedings of the Third European Academy of Forensic Science Triennial Meeting, Istanbul, Turkey, p. 84, 2003.

3. S. N. Srihari, B. Zhang, C. Tomai, S. Lee, Z. Shi, and Y. C. Shin, "A system for hand-writing matching and recognition," Proceedings of the Symposium on Document Image Understanding Technology (SDIUT), Greenbelt, MD, 2003.

4. R. Huber and A. Headrick, Handwriting Identification: Facts and Fundamentals. CRC Press, 1999.

5. G. Srikantan, S. Lam, and S. N. Srihari, "Gradient-based contour encoding for character recognition," Pattern Recognition, vol. 7, pp. 1147-1160, 1996.

6. B. Zhang and S. N. Srihari, "Analysis of handwriting individuality using handwritten words," Proceedings of the Seventh International Conference on Document Analysis and Recognition, Edinburgh, Scotland, 2003.

7. B. Zhang, S. N. Srihari, and S.-J. Lee., "Individuality of handwritten characters," Proceedings of the Seventh International Conference on Document Analysis and Recognition (ICDAR), pp. 1086-1090, 2003.

8. A. S. Osborn, Questioned Documents. Nelson Hall Pub., 1929.

9. C. Champod, "The inference of identity of source: Theory and practice," The First International Conference On Forensic Human Identification In The Millennium, London, UK, pp. 24-26, October 1999.

10. C. F. Tippett, V. J. Emerson, M. J. Fereday, F. Lawton, and S. M. Lampert, "The evidential value of the comparison of paint flakes from sources other than vehicules," Journal of Forensic Sciences Society, vol. 8, pp. 61-65, 1968. 
11. R. Plamondon and S. N. Srihari, "On-line and off-line handwriting recognition: A comprehensive survey," IEEE Transactions on Pattern Analysis and Machine Intelligence, vol. 22, no. 1, pp. 63-84, January 2000.

12. R. Plamondon and G. Lorette, "On-line and off-line handwriting recognition: A comprehensive survey," IEEE Transactions on Pattern Analysis and Machine Intelligence, vol. 22, no. 1, pp. 63-84, January 2000.

13. M. K. Kalera, B. Zhang, and S. N. Srihari, "Off-line signature verification and identification using distance statistics," Proceedings of the International Graphonomics Society Conference, Scottsdale, AZ, pp. 228-232, November 2003.

14. B. Horn, "Robot vision," MIT Press, 1986.

15. M.E.Munich and P.Perona, "Visual identification by signature tracking," IEEE Transactions on Pattern Analysis and Machine Intelligence, vol. 25, no. 2, pp. 200-217, February 2003.

16. B. Fang, C. H. Leung, Y. Y. Tang, K. W. Tse, P. C. K. Kwok, and Y. K. Wong, "Off-line signature verification by the tracking of feature and stroke positions," Pattern Recognition, vol. 36, pp. 91-101, 2003.

17. S. Lee and J. C. Pan, "Off-line tracing and representation of signatures," IEEE Transactions on Systems, Man and Cybernetics, vol. 22, pp. 755-771, 1992.

18. R. Sabourin and R. Plamondon, "Preprocessing of handwritten signatures from image gradient analysis," Proceedings of the 8th International Conference on Pattern Recognition, pp. 576-579, 1986.

19. C. C. Lin and R. Chellappa, "Classification of partial 2-d shapes using fourier descriptors," IEEE Transactions on Pattern Analysis and Machine Intelligence, vol. 9, pp. 696-690, 1997.

20. M. Ammar, Y. Yoshido, and T. Fukumura, "A new effective approach for offline verification of signatures by using pressure features," Proceedings of the 8th International Conference on Pattern Recognition, pp. 566-569, 1986.

21. J. K. Guo, D. Doermann, and A. Rosenfeld, "Local correspondence for detecting random forgeries," Proceedings of the International Conference on Document Analysis and Recognition, pp. 319-323, 1997.

22. A. Xu, M. K. Kalera, and S. N. Srihari, "Learning strategies and classification methods for off-line signature verification," Proceedings of the Ninth International Workshop on Frontiers in Handwriting Recognition, pp. 161-166, October 2004.

23. B. Zhang and S. Srihari, "Binary vector dissimilarity measures for handwriting identification," Proceedings of SPIE, Document Recognition and Retrieval X, pp. 155-166, 2003.

24. T. Joachims, "Text categorization with support vector machines: Learning with many relevant features," Proceedings of the European Conference on Machine Learning, pp. 137-142, 1998.

25. E. Osuna, R. Freund, and F. Girosi, "Support vector machines: Training and applications, Tech. Rep. AIM-1602, MIT, 1997.

26. B. Boser, I. Guyon, and V. Vapnik, "A training algorithm for optimal margin classifiers," Proceedings of the Fifth Annual Workshop on Computational Learning Theory, 1992. 